\title{
Combining transcranial direct current stimulation and gravity- supported, computer-enhanced arm training in a chronic pediatric stroke survivor: a case report
}

\author{
Sofia Straudi ${ }^{1,2}$, Effie Chew ${ }^{1}$, Cajigas Iahn ${ }^{3}$, Felipe Fregni ${ }^{4 *}$ and Paolo Bonato ${ }^{1,3 *}$ \\ ${ }^{1}$ Motion Analysis Laboratory, Department of Physical Medicine and Rehabilitation, Spaulding Rehabilitation Hospital, Harvard Medical School, Boston, MA, USA \\ ${ }^{2}$ Department of Physical Medicine and Rehabilitation, Ferrara University Hospital, Ferrara, Italy \\ ${ }^{3}$ Harvard-MIT Division of Health Sciences and Technology, Cambridge, MA, USA \\ ${ }^{4}$ Spaulding Center of Neuromodulation, Spaulding Rehabilitation Hospital, Harvard Medical School, Boston, MA, USA
}

\begin{abstract}
Introduction: Transcranial direct current stimulation (tDCS) is a non-invasive method of brain stimulation that modulates cortical excitability via constant weak electric currents.

Case presentation: We tested the feasibility and preliminary effects of combined tDCS with repetitive, task-specific practice of the affected upper extremity in a 19-year-old individual with no meaningful use of the right arm due to a chronic left middle cerebral artery infarction at the age of 12 years old. The subject underwent 10 training sessions over 2 weeks during which he received 40 minutes of tDCS while performing repetitive, multidirectional arm reaching and hand grasp/release activities in a gravity-supported, computer-enhanced environment. Potential side effects were investigated using the Digit Span test, the nine-item Patient Health Questionnaire, and the tDCS Adverse Effects Questionnaire. Clinical outcome measures were gathered pre-training, post-training and 2 weeks after the end of training. These measures included (i) the Jebsen-Taylor Hand Function Test, (ii) the Box and Block Test, (iii) the Fugl-Meyer Assessment for upper extremity, and (iv) Motor Activity Log. In addition, during the training sessions, we collected kinematic measures during arm reaching. The subject tolerated the brain stimulation well. There were no effects on mood or working memory. Clinical outcome measures showed improvements in upper extremity motor function that were retained 2 weeks after completion of training. Movement kinematic parameters showed similar trends in response to training: improvements in mean speed, maximum speed, and acceleration of arm reaching movements.
\end{abstract}

Conclusions: Despite the fact that this case report does not detangle the effects of tDCS from motor training, it provides initial detailed clinical and kinematic data showing beneficial effects of tDCS for enhancing motor recovery when combined with Gravity-Supported, Computer-Enhanced Arm Training that are useful for future trials planning the use of this combined approach in pediatric stroke. We review the use of combined robotic-based arm training and tDCS for motor recovery in stroke.

\section{Introduction}

Stroke in children is rare, with a reported incidence of about 2.3 to 13.0 per 100,000 per year [1]. However, up to $45 \%$ of these children continue to experience long-term motor impairments following stroke. Clinically meaningful recovery of the upper limb motor function in the chronic phase is rare despite the plasticity of the developing brain $[2,3]$. Non-invasive brain stimulation is a novel approach that holds the promise of facilitating motor recovery after pediatric stroke. Kirton et al. [4] provided preliminary evidence that repetitive transcranial magnetic stimulation (rTMS) can be used to facilitate the achievement of motor improvements in pediatric stroke survivors via inhibition of the unaffected motor cortex. Transcranial direct current stimulation (tDCS) is another non-invasive brain stimulation technique with potential for improving motor gains. It has advantages over rTMS, such as the greater portability and lower cost, the ability to stimulate both hemispheres simultaneously $[5,6]$, the long-lasting effects on cortical excitability with no significant adverse effects, and the lower level of discomfort experienced by patients. However, tDCS has never been tested in pediatric stroke survivors.

In the single-case study herein presented, we explored the effects of
tDCS in combination with motor training. The goal of the combined treatment is based on the mechanisms of tDCS as this technique changes the neuronal spontaneous firing in a large neuronal network; thus the combination with a behavioral training, such as motor arm training, can guide plasticity and induce significant neuroplastic modifications in the affected corticospinal system. We used tDCS with a montage that allowed inhibition of the unaffected motor cortex (i.e. unaffected M1) via cathodal stimulation and facilitation of the affected motor cortex (i.e. affected M1) via anodal stimulation as used before [6]. We chose a rehabilitation technique marked by high intensity

Correspondence to: Paolo Bonato, PhD, 300 1st Avenue, Navy Yard, Charlestown, Tel: (617) 573 2770; Fax: (617) 573 2769; E-mail: pbonato@partners.org

Felipe Fregni, MD, PhD, 96 13th Street, Charlestown, MA 02129, E-mail: Felipe.Fregni@ppcr.hms.harvard.edu

Key words: transcranial direct current stimulation, upper extremity rehabilitation, pediatric stroke

Received: November 17, 2015; Accepted: December 21, 2015; Published: December 24, 2015 
and specificity of motor training. This choice was motivated by the growing body of evidence indicating that intensity and specificity of motor training are important elements to achieve recovery of motor functions [7-9]. We delivered high intensity and specificity of motor training by using the Armeo Spring system (Hocoma AG). The system is an adjustable arm exoskeleton with integrated springs that can be set to passively counterbalance the weight of the arm, thereby reducing the effort required by the paretic arm to overcome gravity. The device is able to provide visual feedback through a virtual environment (i.e. computer games). Housman et al. [10] showed that the use of the Armeo Spring system in stroke rehabilitation leads to significant motor gains. We used this device to achieve repetitive training of arm reach and hand grasp/release in an engaging environment that could be adapted to the subject capabilities. A data glove system was added to the Armeo system to enhance training of hand grasp/release [11].

Tests administered pre-training, post-training, and 2 weeks after completion of training allowed us to evaluate safety and feasibility of administering the technique in a pediatric chronic stroke survivor. In addition, clinical measures were gathered to assess the initial efficacy of tDCS in combination with motor training. Finally, we collected kinematic measures during training to explore the relationship between motor gains and changes in the biomechanics of movement during training.

\section{Case presentation}

The subject was a 19-year-old male with right hemiparesis due to a left middle cerebral artery territory infarction following spontaneous dissection of the internal carotid artery sustained when he was 12 years old. He had grade 4 strength in the arm and hand as assessed using the Medical Research Council (MRC) Scale for Muscle Strength. The sum of the Modified Ashworth scores for the elbow, wrist and finger flexors and extensors was 8 at baseline. Motor function in the right upper extremity was impaired such that the subject rarely used it in functional tasks during the performance of activities of daily living. $\mathrm{He}$ walked independently and language and cognitive abilities were within normal limits.

Written informed consent was obtained from the patient before the start of the study. After consenting and recruitment in the study, the subject underwent 10 sessions of tDCS concurrent with repetitive, multidirectional arm reaching and hand grasp/release activities in the gravity-supported, computer-enhanced environment provided by the Armeo Spring system (Hocoma AG). Motor training consisted of a variety of motor tasks performed in a virtual environment including: picking up objects from shelves and placing them in a bin, picking up eggs from a basket and cracking them over a frying pan, and picking up apples at a virtual supermarket and placing them in a shopping cart. During training, the subject wore a data glove that allowed us to track hand aperture [11]. The data glove was used to integrate hand grasp/ release movements in the computer games (Figure 1). Training was carried out over 2 weeks.

tDCS was applied over the primary motor cortex (M1) for 40 minutes during each motor training sessions. A continuous current of $1 \mathrm{~mA}$ was applied during training with a ramp up and ramp down time of $10 \mathrm{~s}$. The direct current was generated by a battery-driven, constant current stimulator (Iomed Phoresor PM850) and delivered to the subject by means of a saline-soaked pair of surface sponge electrodes $\left(35 \mathrm{~cm}^{2}\right)$. The positive electrode (i.e. anode) was placed over the affected primary motor cortex (i.e. affected M1). The negative electrode (i.e. cathode) was placed over the contralateral primary motor cortex (i.e.

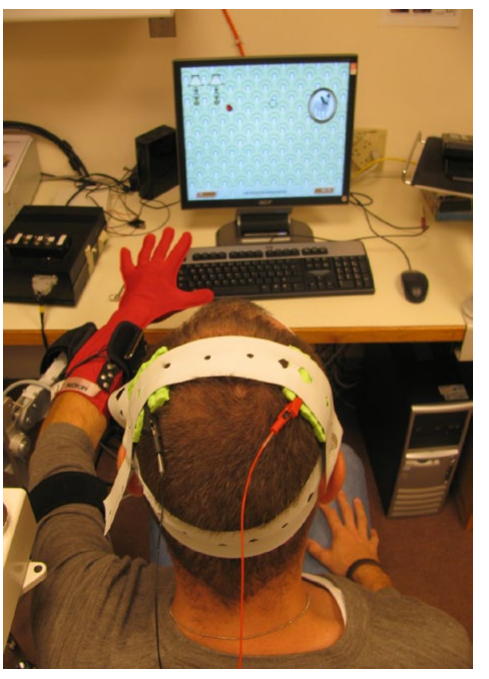

Figure 1. An explicative subject during performance of the assessment game with the Armeo Spring system combined with the data glove. The picture shows also the electrodes used to deliver tDCS during training.

unaffected M1). Electrodes were located at $\mathrm{C} 3$ and $\mathrm{C} 4$ according to the $10 / 20$ international EEG system. The goal of this montage is to decrease cortical excitability in the unaffected motor cortex and increase it in the affected motor cortex as demonstrated before [6].

The subject tolerated the brain stimulation well. No adverse effects as well as effects on mood and working memory were detected as measured by the tDCS Adverse Event Questionnaire, the Patient Health Questionnaire-9, and the forward and backward Digit Span test [12]. tDCS Adverse Event Questionnaire compiled by the subject at the end treatment showed minor scalp discomfort and trouble concentrating, but no headache, neck pain, tingling, scalp burns, skin redness, sleepiness and mood changes. No adverse effects were selfreported by the subject at the 2 -week follow-up visit. No changes were observed in the forward and backward Digit Span test. The subject was able to recall 5 digits forward and 3 digits backward at each testing session. Finally, the Patient Health Questionnaire-9 score remained unchanged to 8 pre-training vs. post-training.

Clinical outcome measures (Table 1) were gathered at pre-training, post-training and 2 weeks after the end of training. Both the Jebsen Taylor Hand Functional Test (JTHFT), a timed test that evaluates 7 hand functions commonly used in activities of daily living, and the Fugl-Meyer Assessment (FMA) test for upper extremity, a measure of motor impairment, improved immediately post training. The subject continued to show improvements in these measures at the 2-week follow-up testing session. Specifically, the subject improved in all the hand tasks of the JTHFT, but more markedly in picking up small objects (paper clips, pennies and bottle tops) and placing them in a can (from $16 \mathrm{~s}$ to $11.9 \mathrm{~s}$ post-training and to $9.3 \mathrm{~s}$ at the 2-week followup testing session). The results of the FMA tests showed gains both in proximal and in distal function. 5-point and 6-point gains with respect to baseline were observed in proximal function post-training and 2-week after completion of training respectively. 3-point and 5-point gains were observed in distal function at the post-training and followup sessions. The Box and Block test, a timed test that measures the number of wooden blocks taken at a time and placed in an empty box in $1 \mathrm{~min}$, showed only minor changes. The Modified Ashworth Scale (MAS) was used to assess spasticity at the elbow, wrist and proximal interphalangeal joints (PIPJ). The sum of the MAS scores for the elbow, 
wrist and PIPJ flexors and extensors was used to track longitudinal changes in the severity of spasticity [13]. Immediately post-training, the above-defined cumulative MAS score decreased from 8 to 5.5. The improvement was retained at the 2-week follow-up session. The Motor Activity Log (MAL), a self-reported measure of the amount and quality of the use of the affected hand in everyday life, showed improvements in both dimensions (i.e. amount of quantity of use) post-training that was retained 2 weeks after completion of training.

Kinematic measures of arm reaching were collected from the Armeo Spring system using a game specifically designed for assessment purposes. In the game, the subject was encouraged to collect coins as they appeared on different parts of the screen. The cursor position on the screen was determined by the $\mathrm{X}$ and $\mathrm{Y}$ coordinates of the distal end of the arm exoskeleton, which corresponded to the medio-lateral and antero-posterior position of the distal end of the exoskeleton respectively. These measures corresponded to an approximation of the $(\mathrm{X}, \mathrm{Y})$ coordinates of the position of the hand in a reference system with origin on the acromion of the ipsilateral shoulder. The $\mathrm{X}$ and $\mathrm{Y}$ position of the distal end of the exoskeleton were remapped in the game in the horizontal and vertical position of a cursor shown on the computer screen. These measures were recorded at $100 \mathrm{~Hz}$ while the subject participated in the assessment game. Although the system provided also the vertical coordinate of the distal end of the arm exoskeleton (i.e. $\mathrm{Z}$ coordinate), we considered the kinematics of the game movements in the $\mathrm{X}, \mathrm{Y}$ plane because only the $\mathrm{X}$ and $\mathrm{Y}$ coordinates of the distal end of the exoskeleton were used in the game. We derived the magnitude of the 2-D position vector of the distal end of the arm exoskeleton defined as: $r(t)=\sqrt{x(t)^{2}+y(t)^{2}}$. In addition, the mean speed, maximum speed, mean velocity, max velocity along with the jerk metric and speed metric were computed over the course of the assessment game. Consistently with the definition proposed by Rohrer et al. [14], we estimated the jerk metric as the negative mean jerk value divided by the maximum speed value. The jerk metric has units of $1 / \mathrm{s}^{2}$. The speed metric was estimated as the normalized mean speed, i.e. the mean speed value divided by the maximum speed value. Changes during the training period in kinematic parameters are shown in Figure 2. Numerical values gathered during the pre-training and post-training sessions are reported in Table 2. Significant changes $(\mathrm{p}<0.05)$ were observed in mean speed value, maximum speed value, maximum acceleration value, and jerk metric. A trend ( $\mathrm{p}=0.064)$ was also observed in minimum acceleration value. No significant changes were observed in speed metric.

\section{Discussion}

This is the first report of safety and feasibility of combining tDCS and motor training in a chronic pediatric stroke survivor. The subject
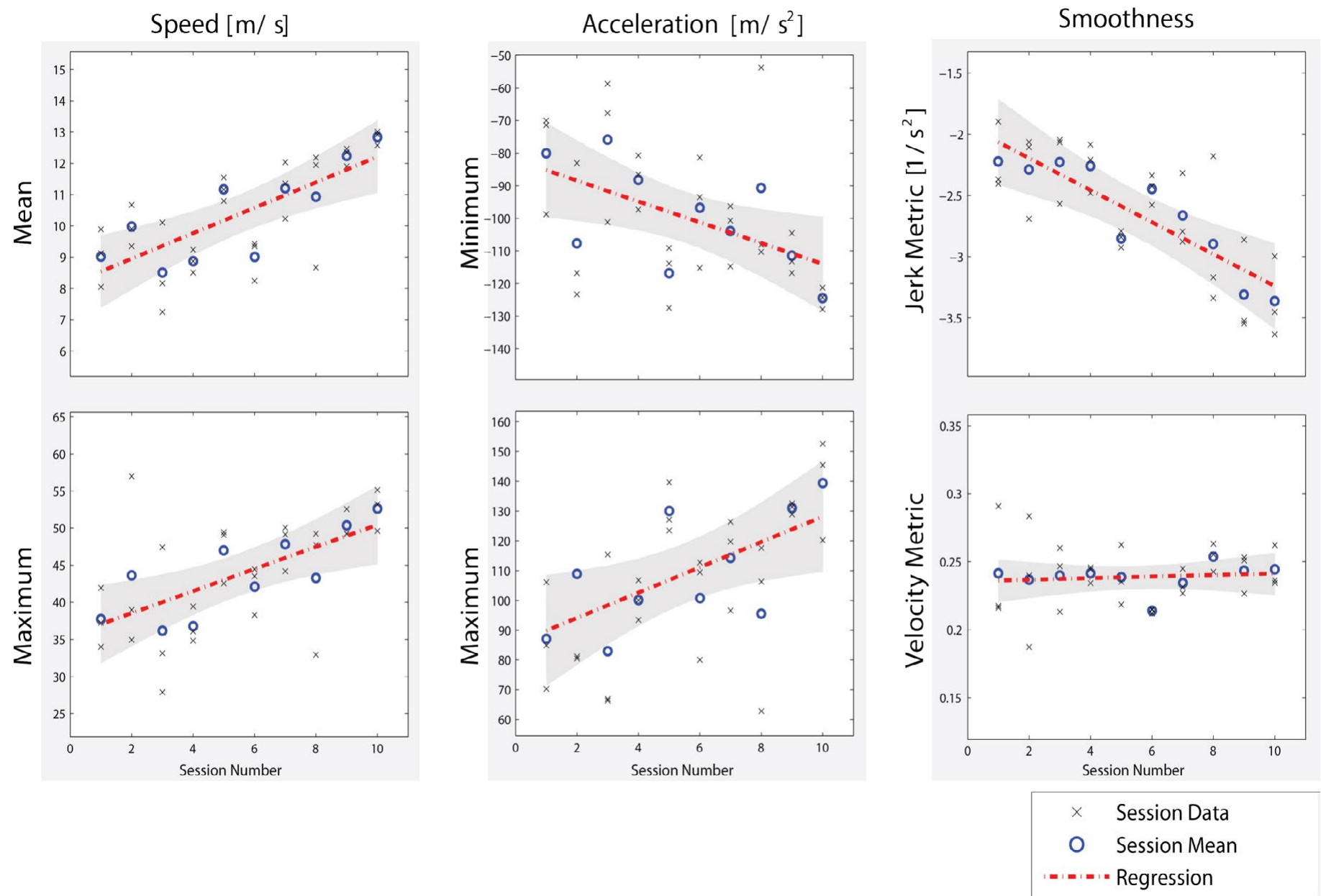

Figure 2. Changes in kinematic measures with training as measured using the assessment game. The game was run 3 times at the end of each training session. Relevant kinematic parameters (i.e. mean speed, maximum speed, minimum acceleration, maximum acceleration, jerk metric, and speed metric) were computed for each run. To assess the effect of individual training sessions on kinematic parameters, a linear regression was performed on the mean kinematic measure values (i.e. circles) for each training session. Statistically significant regression coefficients for the slope of the regression line (summarized in Table 2) suggest a significant change in the kinematic of motion as a function of training. 
Straudi S (2015) Combining transcranial direct current stimulation and gravity-supported, computer-enhanced arm training in a chronic pediatric stroke survivor: a case report

Table 1. Outcome measures at pre-training, post-training and the 2-week follow-up visits.

\begin{tabular}{|l|c|c|c|}
\hline Clinical Test & Pre-training & Post-training \\
\hline Jebsen Taylor Hand Function (s) & 81.8 & 75.8 \\
\hline Box and Block (number) & 34 & 32 & 65 \\
\hline Fugl-Meyer Assessment & 39 & 47 & 36 \\
\hline Modified Ashworth Scale (sum) & 8 & 5.5 & 1.5 \\
\hline Motor Activity Log (amount) & 1.1 & 1.3 & 1.4 \\
\hline Motor Activity Log (quantity) & 1 & 1.3 \\
\hline
\end{tabular}

Table 2. Kinematic measures gathered using the assessment game during the first and last training sessions. Percentage changes and significance of the changes identified by the regression lines are also shown.

\begin{tabular}{|c|c|c|c|c|c|c|}
\hline Parameter & Pre & Post & $\%$ Change & Delta/ Session & Confidence Interval & p-value \\
\hline mean speed & 9.975 & 12.835 & 28.671 & 0.407 & {$[0.1676,0.6464]$} & 0.004 \\
\hline max speed & 43.634 & 52.643 & 20.649 & 1.486 & {$[0.5308,2.4415]$} & 0.007 \\
\hline min accel. & -107.714 & -124.464 & 15.551 & -3.196 & {$[-6.6248,0.2336]$} & 0.064 \\
\hline max accel. & 108.933 & 139.386 & 27.956 & 4.250 & {$[0.3628,8.1374]$} & 0.036 \\
\hline jerk metric & -2.287 & -3.361 & 46.961 & -0.131 & {$[-0.1817,-0.0796]$} & $<0.001$ \\
\hline speed metric & 0.237 & 0.244 & 3.147 & 0.001 & {$[-0.0021,0.0033]$} & 0.637 \\
\hline
\end{tabular}

who participated in the study was a 19-year-old individual with residual hemiparesis following a stroke at the age of 12 years old. The subject tolerated well brain stimulation during the 40 -minute training sessions. He reported minor scalp discomfort and trouble concentrating at the end of treatment (i.e. at the end of the last training session) but no adverse events at 2 weeks after completion of training. Besides, the subject never reported any headache, scalp burning sensation, tingling, skin redness, sleepiness, or mood changes. Furthermore, the Patient Health Questionnaire-9 and the forward and backward Digit Span tests did not show any changes pre-training vs. post-training and at the 2-week follow-up visit.

\section{Limitations and insights of this case report}

The present case report has a number of limitations. First, in a noncontrolled single-case study, placebo effect would be partly responsible for clinical gains. Specifically, placebo effect occurs when subjects expectation leads them to experience some change even though they receive ineffective treatments. Second, our intervention combined two treatments (i.e. real-tDCS and upper extremity motor training); for this reason, our findings could not detangle tDCS effects from arm motor training one.

Besides these limitations, remarkable insights in this report are the kinematic analysis of arm function, in addition to clinical measures. This is a sensitive tool to objectively evaluate impaired function after stroke. Kinematic measures of arm reaching were collected from the Armeo Spring system using a game specifically designed for assessment purposes. The addition of a sensorized glove for hand training may have improved distal recovery and motor skill transfer into daily life activities. Those instruments are closer to achieve the ultimate rehabilitation goal, which is having a real-world effects with positive impact on community-based activites. In addition, it is important to underscore that this subject had undergo several rehabilitation treatments before and had obtained limited improvement in these past interventions. Given the lack of improvement and duration of stroke (7 years), the improvement observed in this case report is promising for future trials testing this combined intervention in pediatric stroke.

\section{Motor training with robotics and virtual environments}

Robotics: In the past decades, many robotic devices have been developed for the upper extremity stroke rehabilitation. Robots may enhance conventional motor therapy, increasing repetitions of well-defined motor tasks (massed practice) with an improvement of motivation due to the feedback of the device; they can be programmed to perform in different functional modes according to the subject level of motor impairment. Existing robotic upper-limb training devices (i.e. MIT-MANUS) [15] primarily train the proximal portions of the upper limb (arm); while few devices provide therapy to the hand and fingers [9]. Takahashi et al. have shown as a robot-based therapy may offer improvements in hand motor function in chronic stroke subjects combined with a cortical reorganization of motor maps. Up to date, several studies highlighted how robot-assisted therapy would improve arm motor function [16-19] and activities of daily living [20] after stroke. In addition, robots can be used to gain insights into the stroke recovery process, such as the anticipatory control of arm movement [21] or motor synergies [22]. Hogan et al. have provided meaningful clinical recommendations on the delivery of upper limb robotic therapy. They have suggested that the form of robotic therapy (active participation, progressive training based on motor coordination) may be more important than its intensity [23].

Virtual reality: Virtual reality training create a simulated environment for the retraining of motor function, through an interacting and motivating setting in which practice intensity and feedback can be tailored on individualized treatments. In virtual reality-based rehabilitation, implicit learning could have a key role leading to functional improvement after stroke. Virtual reality scenario usually reproduces real life objects and actions (i.e. pick up a glass) and could be immersive or non-immersive. Non-immersive systems are more suitable for rehabilitation due to their easier management and lower risk of side effects (i.e. cyber-sickness, dizziness). Interactive gaming systems are new technologies that offer great potential for neurorehabilitation providing high repetition intensity, specific task practice, training in an engaging environment offering high motivation to the patient, and enhanced feedback about the training results and motor task performance. Some controlled studies have been 
published so far exploring the feasibility and effectiveness of VR-based interventions in stroke survivors, and encouraging data are available [24-26].

\section{Gravity-Supported, Computer-Enhanced Arm Training}

The Therapy Wilmington Robotic Exoskeleton (T-WREX) was developed to help subjects who suffered from a brain lesion training their paretic arm [27] through repetitive, task-oriented movements in a virtual learning environment. This is a five freedom device that able arm movements in a wide workspace. Recently, Housman et al. [10] showed that the use of the system in rehabilitation leads to significant motor gains in chronic stroke survivors. By using a data glove in combination with the Armeo Spring system (Hocoma AG), we were able to deliver repetitive training consisting of arm reaching and hand grasp/release movements in an engaging environment, i.e. via the use of computer games. The tasks presented in the computer games were designed with focus on functional movements. Providing external support to the paretic arm (via the system exoskeleton) has been shown to improve the performance of arm reaching tasks in stroke survivors [28]. The effects of the proximal arm gravity - supported environment could encourage a gradually control of voluntary movement (i.e. elbow extension), working against pathological coupling shoulder abduction - elbow flexion evidenced in stroke survivors [28].

\section{Transcranial direct current stimulation (tDCS) for mo- tor recovery in stroke}

Transcranial Direct Current Stimulation (tDCS) is a non-invasive brain stimulation technique that delivers weak direct currents to the cortex via two electrodes placed on the scalp. tDCS acts by inducing sustained changes in neural cell membrane potential: cathodal tDCS leads to brain hyperpolarization (inhibition), whereas anodal results in brain depolarization (excitation) [29,30].

tDCS is a powerful tool to modulate human brain function and to improve the beneficial effects of neurorehabilitation: performance improvements reported so far with tDCS have been moderate in magnitude (10-30\%) and relatively transient. These findings reported motor gains that lasted for more than $30 \mathrm{~min}$ after the end of the stimulation period. Behavioral gains were accompanied by enhanced cortical excitability.

Possible strategies to influence motor function in the paretic hand are providing an up-regulation of excitability in M1 (affected side) via anodal tDCS and a down-regulation of excitability in M1 (unaffected side) via cathodal tDCS.

Anodal tDCS delivered to M1 over the affected side was tested in subjects with chronic stroke in experimental designs [31,32] and recently reviewed [33,34]. Another neuromodulation approach is reducing the excitability in $\mathrm{M} 1$ over the unaffected side with cathodal tDCS [35]. Studies with cathodal tDCS reported improvements in motor task lasted for the same amount of time [36].

Recently, a bilateral tDCS montage has been proposed in stroke survivors to reduce inter-hemispheric inhibition (via cathodal stimulation over M1 unaffected) and to enhance cortical excitability (via anodal stimulation over M1 affected) with the final goal to increase motor function during motor training. This montage seems to be useful to enhance motor performance during Jebsen Taylor Hand Functional Test in a chronic stroke sample [37].

\section{Combined treatment with robotic-based arm training and $\mathrm{tDCS}$}

In animal models combining non-invasive brain stimulation and exercise enhance motor recovery [38]. Few clinical trials have shown the feasibility and effects of combining tDCS and behavioral training. Preliminary data highlighted the positive effects of combining bilateral tDCS and gravity-supported, computer-enhanced arm training in traumatic brain injury survivors [39]. In the past few years, other studies explored the combination of M1 anodal tDCS over the affected hemisphere with robotics during sub-acute [40] and chronic stroke [41] and cathodal tDCS over the unaffected hemisphere with occupational therapy [42].

Recently, a bilateral tDCS montage has been used combined with motor training: this montage seems to be useful to enhance motor effects of physical therapy [43] and constrained-induced movement therapy in stroke survivors [44].

The results of this case report indicate that $\mathrm{tDCS}$ is a promising technique for enhancing motor recovery when combined with repetitive functional task training. After training, both proximal (shoulder and elbow) and distal (wrist and hand) impairment measures gathered using the Fugl-Meyer Assessment scale improved markedly. The motor gains shown by measures of impairments affecting the shoulder and elbow may be attributable to the training of arm reaching activities, while the motor gains at the wrist and hand may be due to the training of hand grasp/release activities using the data glove. Improvements at the wrist and hand were likely enhanced by tDCS, which was applied over the cortical representation of the hand motor area. Changes in impairment level shown by the Fugl-Meyer Assessment measures were paralleled by changes in functional level measured using the Jebsen Taylor Hand Functional Test. Interestingly, the Fugl-Meyer Assessment scale measures and the Jebsen Taylor Hand Functional Test measures showed further clinical improvements at the 2-week follow-up visit compared to post-training. A possible explanation may be that long-lasting neuromodulatory effects of tDCS might have augmented the benefits associated with an increased level of amount and quality of use of the affected side (shown by the Motor Activity Log measures) due to the improved upper extremity functional level reached by the patient after training. In other terms, the greater use of the affected arm in combination with long-lasting effects of tDCS may have led to further improvements during the two weeks following completion of training.

Consistently with previous reports in adult stroke survivors, we speculate that motor training and tDCS might mutually increase their effects thus leading to significant improvements in motor skills $[31,32]$. The aim of combining tDCS and motor training is to modulate the response of motor cortex area to a behavioral therapy via the neuromodulation effect of tDCS. So far, encouraging results have been shown in animal experiments [45] and human studies [6,40,41]. The results herein presented add positive findings to previous literature and encourage further studies in pediatric stroke survivors.

The large motor gain observed in this single-case study may be attributable to a combination of factors: the intensity and taskspecificity of the motor training regimen, the neuromodulation effect of tDCS and the young age of the subject which is likely to be associated with increased plasticity compared to adult stroke survivors. These results are particularly remarkable given that the subject underwent motor training seven years after suffering a stroke. 
Straudi S (2015) Combining transcranial direct current stimulation and gravity-supported, computer-enhanced arm training in a chronic pediatric stroke survivor: a case report

\section{Future directions}

Up to date, the best way to optimize the effects of coupling tDCS and motor training still need further investigations. Presumably, important key factors are the time from stroke, the role of lesion site, the site and type of stimulation, the timing of stimulation in relation to physical intervention and the motor task.

tDCS stimulation can be delivered just before the motor task, priming functional networks for the physical intervention; during the behavioral intervention when it might preferentially interact with the networks selectively recruited by the ongoing task; or after motor training to promote a long-term consolidation of new neural pathways. Relative to the parameters of stimulation (number of session, frequency, intensity and site) future clinical trails need to take into account that only prolonged and consecutive sessions of tDCS can translate into a long-lasting functional gains in stroke patients [46].

An area for future investigation is also the detection of motor tasks more susceptible to modulation by tDCS, in order to promote the maximum functional recovery in stroke survivors. Future clinical trials should be designed including neurophysiological (i.e. transcranial magnetic stimulation) and neuroimaging (i.e.functional magnetic resonance) measurements to better understand neural substrates underlying motor performance. The findings of our report motivate future investigations concerning the use of tDCS to enhance the effects of rehabilitation interventions. Future studies would have to assess if a longer training program would result in greater functional benefits. Otherwise, a longer-term follow-up is necessary to evaluate long-term retention of the observed motor gains.

\section{Competing interests}

The authors declare that they have no competing interests.

\section{Authors contributions}

SS, EC, CI collected all the data. All authors analyzed and interpreted the patient data as well contributed significantly for the writing of the manuscript. All authors read and approved the final manuscript.

\section{Acknowledgements}

The authors would like to thank Fabrizio Cutolo, Danilio De Rossi, Rita Paradiso, and Alessandro Tognetti for providing the data glove system used in the project. The authors are grateful to Chiara Mancinelli, Mel Meister, and Shaymal Patel for their technical assistance.

\section{References}

1. Amlie-Lefond C, Sebire G, Fullerton HJ (2008)Recent developments in childhood arterial ischaemic stroke. Lancet Neurol 7:425-35. [Crossref]

2. deVeber GA, MacGregor D, Curtis R, Mayank S (2000) Neurologic outcome in survivors of childhood arterial ischemic stroke and sinovenous thrombosis. $J$ Child Neurol 15: 316-324.[Crossref]

3. Kirton A, Westmacott R, deVeber G (2007) Pediatric stroke: rehabilitation of focal injury in the developing brain. NeuroRehabilitation 22:371-82. [Crossref]

4. Kirton A, Chen R, Friefeld S, Gunraj C, Pontigon AM, et al. (2008) Contralesional repetitive transcranial magnetic stimulation for chronic hemiparesis in subcortical paediatric stroke: a randomised trial. Lancet Neurol 7:507-13. [Crossref]

5. Wagner T, Fregni F, Eden U, Ramos-Estebanez C, Grodzinsky A, et al. (2006) Transcranial magnetic stimulation and stroke: a computer-based human model study. Neuroimage30: 857-870.[Crossref]

6. Williams JA, Pascual-Leone A, Fregni F (2010)Interhemispheric modulation induced by cortical stimulation and motor training. PhysTher 90:398-410. [Crossref]
7. Kwakkel G, Wagenaar RC, Twisk JW, Lankhorst GJ, Koetsier JC (1999) Intensity of leg and arm training after primary middle-cerebral-artery stroke: arandomised trial. Lancet 354:191-6. [Crossref]

8. Blennerhassett J, Dite W (2004) Additional task-related practice improves mobility and upper limb function early after stroke: a randomised controlled trial. Aust $J$ Physiother 50: 219-224.[Crossref]

9. Takahashi CD, Der-YeghiaianL, Le V, Motiwala RR, Cramer SC (2008) Robot-based hand motor therapy after stroke. Brain131(Pt 2):425-37. [Crossref]

10. Housman SJ, Scott KM, Reinkensmeyer DJ (2009)A randomized controlled tria of gravity-supported, computer-enhanced arm exercise for individuals with severe hemiparesis. Neurorehabil Neural Repair 23: 505-14. [Crossref]

11. Cutolo F,Mancinelli C, Patel S, Carbonaro N, Schmid M, et al. (2009) "A Sensorized Glove for Hand Rehabilitation". 35th Annual Northeast Bioengineering Conference; April 3- 5, 2009; Cambridge, MA2009.

12. Poreisz C, Boros K, Antal A, Paulus W (2007) Safety aspects of transcranial direct current stimulation concerning healthy subjects and patients. Brain Res Bull 72: 208214.[Crossref]

13. Alibiglou L, Rymer WZ, Harvey RL, Mirbagheri MM (2008) The relation between Ashworth scores and neuromechanical measurements of spasticity following stroke. $J$ NeuroengRehabil 5: 18.[Crossref]

14. Rohrer B, Fasoli S, Krebs HI, Hughes R, Volpe B, et al. (2002) Movement smoothness changes during stroke recovery. J Neurosci22: 8297-8304.[Crossref]

15. Shadmehr R, Mussa-Ivaldi FA (1994) Adaptive representation of dynamics during learning of a motor task. J Neurosci14: 3208-3224.[Crossref]

16. Krebs H, Hogan N, Volpe BT, Aisen ML, Edelstein L et al. (1999) Overview of clinical trials with MIT-MANUS: a robot-aided neuro-rehabilitation facility. Technol Health Care 7:419-23. [Crossref]

17. Lum PS, Burgar CG, Shor PC, Majmundar M, Van der Loos M (2002) Robotassisted movement training compared with conventional therapy techniques for the rehabilitation of upper-limb motor function after stroke. Arch Phys Med Rehabil 83: 952-959.[Crossref]

18. Volpe BT, Krebs HI, Hogan N, Edelsteinn L, Diels CM, et al. (1999) Robot training enhanced motor outcome in patients with stroke maintained over 3 years. Neurology 53: 1874-1876.[Crossref]

19. Fasoli SE, Krebs HI, Stein J, Frontera WR, Hogan N (2003) Effects of robotic therapy on motor impairment and recovery in chronic stroke. Arch Phys Med Rehabil 84:47782. [Crossref]

20. Mehrholz J, Hadrich A, Platz T, Kugler J, Pohl M (2012) Electromechanical and robotassisted arm training for improving generic activities of daily living, arm function, and arm muscle strength after stroke. Cochrane Database Syst Rev 6:CD006876. [Crossref]

21. Takahashi CD, Reinkensmeyer DJ (2003) Hemiparetic stroke impairs anticipatory control of arm movement. Exp Brain Res 149: 131-140.[Crossref]

22. Dipietro L, Krebs HI, Fasoli SE, Volpe BT, Stein J, et al. (2007) Changing motor synergies in chronic stroke. J Neurophysiol 98: 757-68. [Crossref]

23. Hogan N, Krebs HI, Rohrer B, Palazzolo JJ, Dipietro L, et al. (2006) Motions or muscles? Some behavioral factors underlying robotic assistance of motor recovery. $J$ Rehabil Res Dev 43: 605-618.[Crossref]

24. Piron L, Tonin P, Atzori AM, Zucconi C, Massaro C, et al. (2003) The augmentedfeedback rehabilitation technique facilitates the arm motor recovery in patients after a recent stroke. Stud Health Technol Inform 94:265-7. [Crossref]

25. Merians AS, Jack D, Boian R, Tremaine M, Burdea GC, et al. (2002) Virtual realityaugmented rehabilitation for patients following stroke. PhysTher 82: 898-915. [Crossref]

26. Laver K, George S, Thomas S, Deutsch JE, Crotty M (2012) Cochrane review: virtual reality for stroke rehabilitation. Eur J PhysRehabil Med 48:523-30. [Crossref]

27. Rahman T, Sample W, Jayakumar S, King MM, Wee JY, et al. (2006) Passive exoskeletons for assisting limb movement. J Rehabil Res Dev 43: 583-590.[Crossref]

28. Beer RF, Ellis MD, Holubar BG, Dewald JP (2007) Impact of gravity loading on poststroke reaching and its relationship to weakness. Muscle Nerve 36: 242-250.[Crossref]

29. Nitsche MA, Fricke K, Henschke U, Schlitterlau A, Liebetanz D, et al. (2003) Pharmacological modulation of cortical excitability shifts induced by transcranial direct current stimulation in humans. J Physiol553: 293-301.[Crossref] 

a case report

30. Nitsche MA, Doemkes S, Karaköse T, Antal A, Liebetanz D, et al. (2007) Shaping the effects of transcranial direct current stimulation of the human motor cortex. $J$ Neurophysiol97: 3109-3117.[Crossref]

31. Boggio PS, Castro LO, Savagim EA, Braite R, Cruz VC, et al. (2006) Enhancement of non-dominant hand motor function by anodal transcranial direct current stimulation. NeurosciLett 404:232-6. [Crossref]

32. Hummel F, Celnik P, Giraux P, Floel A, Wu WH, et al. (2005) Effects of non-invasive cortical stimulation on skilled motor function in chronic stroke. Brain 128: 490-499. [Crossref]

33. Bastani A, Jaberzadeh S (2012)Does anodal transcranial direct current stimulation enhance excitability of the motor cortex and motor function in healthy individuals and subjects with stroke: a systematic review and meta-analysis. ClinNeurophysiol 123: 644-57. [Crossref]

34. Nowak DA, Bösl K, Podubeckà J, Carey JR (2010) Noninvasive brain stimulation and motor recovery after stroke. RestorNeurolNeurosci 28: 531-544.[Crossref]

35. Murase N, Duque J, Mazzocchio R, Cohen LG (2004) Influence of interhemispheric interactions on motor function in chronic stroke. Ann Neurol55: 400-409.[Crossref]

36. Fregni F, Boggio PS, Mansur CG, Wagner T, Ferreira MJ, et al. (2005) Transcranial direct current stimulation of the unaffected hemisphere in stroke patients. Neuroreport 16:1551-5. [Crossref]

37. Mahmoudi H, BorhaniHaghighi A, Petramfar P, Jahanshahi S, Salehi Z, et al. (2011) Transcranial direct current stimulation: electrode montage in stroke. DisabilRehabil 33: 1383-1388.[Crossref]

38. Teskey GC, Flynn C, Goertzen CD, Monfils MH, Young NA (2003) Cortica stimulation improves skilled forelimb use following a focal ischemic infarct in the rat. Neurol Res 25:794-800. [Crossref]
39. Chew E, Straudi S, Fregni F, Mancinelli C, Patel S, et al. (2009) The effect of combined transcranial direct current stimulation and repetitive functional task training on motor recovery in traumatic brain injury survivors. 5thWorld Congress of the International Society of Physical and Rehabilitation Medicine; June 2009. p. 13-7.

40. Hesse S, Werner C, Schonhardt EM, Bardeleben A, Jenrich W, et al. (2007) Combined transcranial direct current stimulation and robot-assisted arm training in subacute stroke patients: a pilot study. RestorNeurolNeurosci 25:9-15. [Crossref]

41. Edwards DJ, Krebs HI, Rykman A, Zipse J, Thickbroom GW, et al. (2009) Raised corticomotor excitability of M1 forearm area following anodal tDCS is sustained during robotic wrist therapy in chronic stroke. RestorNeurolNeurosci27:199-207. [Crossref]

42. Nair DG, Renga V,Lindenberg R, Zhu L, Schlaug G (2011)Optimizing recovery potential through simultaneous occupational therapy and non-invasive brainstimulation using tDCS. RestorNeurolNeurosci 29: 411-20. [Crossref]

43. Lindenberg R, Zhu LL, Schlaug G (2012) Combined central and peripheral stimulation to facilitate motor recovery after stroke: the effect of number of sessions on outcome. Neurorehabil Neural Repair 26: 479-83. [Crossref]

44. Bolognini N, Vallar G, Casati C, Latif LA, El-Nazer R, Williams J, et al. (2011) Neurophysiological and behavioral effects of tDCS combined with constraint-induced movement therapy in poststroke patients. Neurorehabil Neural Repair25:819-29. [Crossref]

45. Plautz EJ, Milliken GW, Nudo RJ (2000) Effects of repetitive motor training on movement representations in adult squirrel monkeys: role of use versus learning. Neurobiol Learn Mem74: 27-55.[Crossref]

46. Bolognini N, Pascual-Leone A, Fregni F (2009) Using non-invasive brain stimulation to augment motor training-induced plasticity. J NeuroengRehabil 6: 8.[Crossref]

Copyright: (2015 Straudi S. This is an open-access article distributed under the terms of the Creative Commons Attribution License, which permits unrestricted use, distribution, and reproduction in any medium, provided the original author and source are credited. 